\title{
Asymptomatic deep vein thromboses in prolonged hospitalized COVID-19 patients
}

\author{
Marko Lucijanic (D) - Nevenka Piskac Zivkovic - Marija Ivic - Martina Sedinic - Boris Brkljacic - Andrea Mutvar • \\ Armin Atic · Diana Rudan · Bruno Barsic · Ivica Luksic · Rajko Kusec • Gordana Ivanac
}

Received: 22 April 2021 / Accepted: 18 October 2021 / Published online: 25 November 2021

(C) The Author(s), under exclusive licence to Springer-Verlag GmbH Austria, part of Springer Nature 2021

\begin{abstract}
Summary High incidence of venous thromboembolic (VTE) events in coronavirus disease 2019 (COVID-19) patients has been reported despite pharmacologic thromboprophylaxis. We performed prospective bilateral lower extremity ultrasound evaluation of prolonged hospitalized COVID-19 ward patients from our institution without clinical suspicion of deep vein thrombosis (DVT).

A total of 102 patient were included in the study. All patients were receiving pharmacologic thromboprophylaxis, the majority in intermediate or therapeutic doses. Asymptomatic DVT was detected in 26/102 (25.5\%) patients: 22 had distal and four had proximal DVT, six had bilateral leg involvement. Pulmonary embolism was highly prevalent (17/70, 24.3\%) but similarly grouped among patients with and without asymptomatic DVT. In total $37.2 \%$ of patients included in the study were recognized as having VTE.
\end{abstract}

\section{Supplementary Information The online version of this article (https://doi.org/10.1007/s00508-021-01973-1) contains supplementary material, which is available to authorized users.}

M. Lucijanic, MD PhD $(\varangle) \cdot$ M. Ivic $\cdot$ M. Sedinic $\cdot$ R. Kusec Hematology Department, Dubrava University Hospital, Av. Gojka Suska 6, 10000 Zagreb, Croatia

markolucijanic@yahoo.com

M. Lucijanic, MD PhD · M. Ivic · M. Sedinic · A. Mutvar · A. Atic · D. Rudan · B. Barsic · I. Luksic · R. Kusec Primary respiratory and intensive care center, Dubrava University Hospital, Zagreb, Croatia

M. Lucijanic, MD PhD · N. Piskac Zivkovic · B. Brkljacic B. Barsic · I. Luksic · R. Kusec · G. Ivanac

School of Medicine, University of Zagreb, Zagreb, Croatia

N. Piskac Zivkovic

Pulmology Department, Dubrava University Hospital,

Zagreb, Croatia
Asymptomatic DVT events were more common in intensive care unit (ICU) survivors $60 \%$ in postmechanically ventilated ICU survivors, $21.2 \%$ in ward patients, $22 \%$ in high-flow oxygen treated patients; $P=0.031$ ), in patients with higher modified International Medical Prevention Registry on Venous Thromboembolism (IMPROVE) VTE risk-score (median 3 vs. 2 points with and without DVT; $P=0.021$ ) and higher body temperature on admission (median $38.7^{\circ} \mathrm{C}$ vs. $37.7^{\circ} \mathrm{C}$ with and without DVT; $P=0.001$ ). No clear associations with Padua VTE risk score, demographic and other clinical characteristics, intensity of thromboprophylaxis, severity of other COVID-19 symptoms, degree of systemic inflammation or D-dimers on admission were found ( $P>0.05$ for all analyses).

Systematic ultrasound assessment in prolonged hospitalized severe COVID-19 patients prior to hospital discharge is needed, especially in ICU survivors, to

\section{B. Brkljacic · G. Ivanac}

Department of Diagnostic and Interventional Radiology, Dubrava University Hospital, Zagreb, Croatia

\section{A. Mutvar}

Department of Nuclear Medicine, Dubrava University Hospital, Zagreb, Croatia

D. Rudan

Cardiology Department, Dubrava University Hospital,

Zagreb, Croatia

University North, University Centre Varazdin, Varazdin, Croatia

Catholic University of Croatia, Zagreb, Croatia

I. Luksic

Department of Maxillofacial Surgery, Dubrava University Hospital, Zagreb, Croatia 
timely recognize and appropriately treat patients with asymptomatic DVT.

Keywords Acquired coagulation disorders · SARS$\mathrm{CoV}-2 \cdot$ Duplex ultrasound $\cdot$ Screening

\section{Introduction}

Coronavirus disease 2019 (COVID-19) caused by the severe acute respiratory syndrome coronavirus 2 (SARS-CoV-2) presents with severe or critical respiratory symptoms (e.g. pneumonia with hypoxemia) in about $20 \%$ of affected individuals [1].

Patients suffering from severe COVID-19 may develop a spectrum of coagulation abnormalities associated with hypercoagulable state, such as elevated D-dimers, increased factor VIII activity and increased fibrinogen concentration [2, 3]. Frequency of venous thromboembolic events (VTE), including deep vein thrombosis (DVT) and pulmonary embolism (PE), that develop despite using prophylactic anticoagulation varies depending on severity of COVID-19 in investigated cohorts and whether only symptomatic clinical events were considered. A large study with over 3000 hospitalized patients reported incidence of $3.2 \%$ for PE and $3.9 \%$ for DVT in an overall cohort, $6.2 \%$ and $9.4 \%$ in intensive care unit (ICU), and $2.2 \%$ and $2.0 \%$ in non-ICU patients, respectively [4]; however, studies that performed systematic DVT assessment by bilateral leg ultrasound reported high frequency of mostly asymptomatic DVT in the range 11.9-22.5\% among hospitalized ward patients [5-7] and $65-69 \%$ among ICU patients on admission $[8,9]$.

Patients with severe symptoms of COVID-19 often require prolonged hospitalization with reduced mobility due to both imposed restrictions (dependence on oxygen source) and objective decline in functional status (hypoxia, systemic inflammation, muscle wasting ...). Prompted by literature reports of high asymptomatic VTE prevalence in non-ICU and ICU COVID-19 patients, several sudden deaths of patients who survived ICU and returned to the wards and unpublished experience from our post-COVID outpatient clinic, we decided to assess the prevalence of DVT among prolonged hospitalized COVID19 patients without clinical suspicion of DVT and to investigate potential predictors.

\section{Patients and methods}

During January and February 2021 we have prospectively undertaken bilateral lower extremity deep vein duplex ultrasound evaluation in 102 consecutive COVID-19 patients without clinical suspicion of DVT who were hospitalized in our institution for a prolonged period. Our institution is a dedicated COVID19 hospital, completely repurposed for the treatment of COVID-19 patients, treating mostly patients with severe or critical symptoms requiring oxygen supple- mentation treatment. Patients considered eligible for the study were adult ( $\geq 18$ years) ward patients who required hospitalization for $\geq 7$ days regardless whether they previously received ICU level care due to respiratory instability or prior oral anticoagulant therapy use. Diagnosis of COVID-19 was established as a positive result on a real time PCR assay or antigen test of nasopharyngeal swabs. All patients were of White race. All patients were receiving low molecular weight heparin (LMWH) thromboprophylaxis (either by enoxaparin or dalteparin), intensity of which was decided by the discretion of treating physicians and guided by the severity of respiratory instability, perceived bleeding risk and individual preferences. Enoxaparin doses $40 \mathrm{mg}$ once daily and dalteparin doses 5000 units once daily were considered prophylactic, enoxaparin doses $1 \mathrm{mg} / \mathrm{kg}$ twice daily and dalteparin doses 100 units/kg twice daily were considered therapeutic and other in between dosing was considered intermediate. All patients with pneumonia requiring oxygen supplementation were receiving dexamethasone. Baseline clinical and laboratory parameters at the time of hospital admission were recorded. Predetermined DVT risk was assessed using the modified international medical prevention registry on venous thromboembolism [10] (IMPROVE), IMPROVEDD [11] and Padua [12] VTE risk scores. Severity of COVID-19 clinical presentation and prognosis were assessed using the modified early warning score [13] (MEWS) and 4C mortality score [14]. Patients were examined for DVT of lower extremities using the LOGIQ E9 state of the art ultrasound scanner (GE Healthcare, USA). Two linear transducers were used, one $9 \mathrm{MHz}$ frequency transducer, and another matrix 6-12 MHz transducer. Color duplex Doppler examination was performed in all patients on femoral veins, popliteal veins, posterior tibial, anterior tibial, peroneal, gastrocnemic and sural veins, which included detection of flow with color Doppler, compression, and analysis of flow augmentation on distal compression. DVT was considered as distal if affecting infrapopliteal deep veins or as proximal if affecting popliteal or deep thigh veins. Chest CT angiography scan (Somatom Definition AS+, Siemens AG, Erlangen, Germany) or perfusion scintigraphy (NM/CT 850, GE; PULMOCIS, CIS, 99mTc-MAA) were performed in cases of suspected pulmonary embolism. The study was approved by the institutional review board. All patients provided informed consent for study procedures.

\section{Statistical methods}

Normality of distribution of numerical variables was tested using the Shapiro Wilk test. All assessed numerical variables did not demonstrate normal distribution and they were presented as median and interquartile range (IQR) and were compared between the groups using the Mann-Whitney $U$-test. Categorical variables were presented as proportions and 
Table 1 Patient characteristics in an overall cohort and stratified according to the presence of clinically unsuspected deep vein thrombosis (DVT)

\begin{tabular}{|c|c|c|c|c|}
\hline & Overall cohort & No DVT & DVT present & $P$-value \\
\hline Total number & 102 & $76 / 102(74.5 \%)$ & $26 / 102(25.5 \%)$ & \multirow[t]{2}{*}{0.997} \\
\hline Age (years) & 71.5 IQR (64.25-79) & 71.5 IQR (64-79) & 71 IQR (65-78.75) & \\
\hline \multicolumn{5}{|l|}{ Sex } \\
\hline Male & $54 / 102(52.9 \%)$ & $40 / 76(52.6 \%)$ & $14 / 26(53.8 \%)$ & \multirow[t]{2}{*}{0.915} \\
\hline Female & $48 / 102(47.1 \%)$ & $36 / 76(47.4 \%)$ & $12 / 26(46.2 \%)$ & \\
\hline Length of hospitalization at the time of evaluation (days) & 18 IQR (11-23.75) & 19 IQR (9.75-24.5) & 17.5 IQR (13.25-22) & 0.923 \\
\hline Time from onset of symptoms to evaluation (days) & 27 IQR (22-35.75) & 27 IQR (21.75-36.25) & 28 IQR (22.5-34.75) & 0.612 \\
\hline Pneumonia & $97 / 102(95.1 \%)$ & $71 / 76(93.4 \%)$ & $26 / 26(100 \%)$ & 0.325 \\
\hline Oxygen supplementation & $96 / 102(94.1 \%)$ & 70/76 (92.1\%) & $26 / 26(100 \%)$ & 0.334 \\
\hline \multicolumn{5}{|l|}{ Level of pre-evaluation critical care } \\
\hline Prolonged ward hospitalization & $59 / 102(57.8 \%)$ & $46 / 76(60.5 \%)$ & $13 / 26(50 \%)$ & \multirow[t]{3}{*}{0.031 * } \\
\hline Separated from HFOT & $33 / 102(32.4 \%)$ & $26 / 76(34.2 \%)$ & $7 / 26(26.9 \%)$ & \\
\hline Mech. ventilated ICU survivors & $10 / 102(9.8 \%)$ & $4 / 76(5.3 \%)$ & $6 / 26(23.1 \%)$ & \\
\hline \multicolumn{5}{|l|}{ LMWH doses } \\
\hline Prophylactic & $31 / 102(30.4 \%)$ & $26 / 76(34.2 \%)$ & $5 / 26(19.2 \%)$ & \multirow[t]{3}{*}{0.155} \\
\hline Intermediate & $17 / 102(16.7 \%)$ & $14 / 76(18.4 \%)$ & $3 / 26(11.5 \%)$ & \\
\hline Therapeutic & $54 / 102(52.9 \%)$ & $36 / 76(47.4 \%)$ & $18 / 26(69.2 \%)$ & \\
\hline Admission CRP (mg/L) & 108 IQR (48.85-156.6) & 107.8 IQR (48.25-145.83) & 134.7 IQR (49.85-172.75) & 0.232 \\
\hline Admission ferritin $(\mu \mathrm{g} / \mathrm{L})$ & 840.5 IQR (437.75-1439) & 769 IQR (393.25-1439) & 976 IQR (660.25-1321.5) & 0.530 \\
\hline Admission WBC $\left(\times 10^{9} / \mathrm{L}\right)$ & 9.5 IQR (6.8-12.95) & 9.4 IQR $(6.75-13)$ & 9.7 IQR (7.28-11.6) & 0.963 \\
\hline Admission abs. neutrophil count $\left(\times 10^{9} / \mathrm{L}\right)$ & 8 IQR (5.36-10.54) & 8 IQR (5.32-10.71) & 8.1 IQR (5.59-9.95) & 0.872 \\
\hline Admission abs. Iymphocyte count $\left(\times 10^{9} / \mathrm{L}\right)$ & 0.8 IQR $(0.51-1.3)$ & 0.8 IQR (0.52-1.33) & 0.7 IQR (0.4-0.94) & 0.099 \\
\hline Admission platelets $\left(\times 10^{9} / \mathrm{L}\right)$ & 229 IQR (171.25-310) & 227 IQR (171.75-313.25) & 250.5 IQR (161.75-290.75) & 0.851 \\
\hline Admission hemoglobin ( $\mathrm{g} / \mathrm{L})$ & 128 IQR (113-141) & 128.5 IQR (113.75-141) & 127.5 IQR (110.75-139.5) & 0.744 \\
\hline Admission RDW (\%) & 13.9 IQR (13.33-14.95) & 14 IQR (13.35-14.8) & 13.9 IQR (13.33-15.1) & 0.551 \\
\hline Admission D-dimers ${ }^{+}$(mg/L FEU) & 1.7 IQR (0.83-3.96) & 1.6 IQR $(0.76-3.74)$ & 2 IQR (0.96-4.37) & 0.401 \\
\hline \multicolumn{5}{|l|}{ Admission D-dimers (mg/L FEU) } \\
\hline$<1.5$ & $39 / 90(43.3 \%)$ & $28 / 65(43.1 \%)$ & $11 / 25(44 \%)$ & \multirow[t]{3}{*}{0.279} \\
\hline $1.5-3.0$ & $20 / 90(22.2 \%)$ & $17 / 65(26.2 \%)$ & $3 / 25(12 \%)$ & \\
\hline$>3.0$ & $31 / 90(34.4 \%)$ & $20 / 65(30.8 \%)$ & $11 / 25(44 \%)$ & \\
\hline D-dimers control+ (mg/L FEU) & 1.6 IQR (0.74-3.91) & 1.4 IQR $(0.71-3.3)$ & 3.3 IQR (1.31-4.36) & 0.190 \\
\hline \multicolumn{5}{|l|}{ D-dimers control (mg/L FEU) } \\
\hline$<1.5$ & $25 / 55(45.5 \%)$ & 20/37 (54.1\%) & $5 / 18(27.8 \%)$ & \multirow[t]{3}{*}{0.101} \\
\hline $1.5-3.0$ & $10 / 55(18.2 \%)$ & $7 / 37(18.9 \%)$ & $3 / 18(16.7 \%)$ & \\
\hline$>3.0$ & $20 / 55(36.4 \%)$ & $10 / 37(27 \%)$ & $10 / 18(55.6 \%)$ & \\
\hline Charlson comorbidity index & 4 IQR (3-6) & 4 IQR (3-5.25) & $3 \mathrm{IQR}(2.25-6)$ & 0.783 \\
\hline Number of comorbidities & 2 IQR (1-3) & 2 IQR (1.75-3) & 2 IQR (1-3) & 0.457 \\
\hline Previous oral anticoagulant therapy & 9/102 (8.8\%) & $6 / 76(7.9 \%)$ & $3 / 26(11.5 \%)$ & 0.690 \\
\hline Modified IMPROVE score & 2 IQR (1-3) & 2 IQR (1-3) & 3 IQR (2-3.75) & 0.021 * \\
\hline \multicolumn{5}{|l|}{ Modified IMPROVE score } \\
\hline 0-1 point & $30 / 102(29.4 \%)$ & $26 / 76(34.2 \%)$ & $4 / 26(15.4 \%)$ & \multirow[t]{4}{*}{0.031 * } \\
\hline 2 points & $27 / 102(26.5 \%)$ & $21 / 76(27.6 \%)$ & $6 / 26(23.1 \%)$ & \\
\hline 3 points & $26 / 102(25.5 \%)$ & $17 / 76(22.4 \%)$ & 9/26 (34.6\%) & \\
\hline$\geq 4$ points & 19/102 (18.6\%) & $12 / 76(15.8 \%)$ & 7/26 (26.9\%) & \\
\hline IMPROVEDD score & 4 IQR (3-5) & 3 IQR (3-4) & 4 IQR (3-5) & 0.029 * \\
\hline Padua score & 6 IQR (5-7) & 6 IQR (5-7) & 6 IQR (5-7.75) & 0.451 \\
\hline \multicolumn{5}{|l|}{ Padua score } \\
\hline$<4$ points & $12 / 102(11.8 \%)$ & 10/76 (13.2\%) & $2 / 26(7.7 \%)$ & \multirow[t]{2}{*}{0.458} \\
\hline$\geq 4$ points & $90 / 102(88.2 \%)$ & $66 / 76(86.8 \%)$ & $24 / 26(92.3 \%)$ & \\
\hline
\end{tabular}


Table 1 (Continued)

\begin{tabular}{|c|c|c|c|c|}
\hline & Overall cohort & No DVT & DVT present & $P$-value \\
\hline MEWS score & 3 IQR (2-4) & 3 IQR (2-4) & 4 IQR (2-4) & 0.214 \\
\hline \multicolumn{5}{|l|}{ MEWS score } \\
\hline Mild symptoms & $3 / 102(2.9 \%)$ & $3 / 76$ (3.9\%) & $0 / 26(0 \%)$ & \multirow[t]{4}{*}{0.573} \\
\hline Moderate symptoms & $2 / 102(2 \%)$ & $2 / 76(2.6 \%)$ & $0 / 26(0 \%)$ & \\
\hline Severe symptoms & $81 / 102(79.4 \%)$ & $60 / 76(78.9 \%)$ & $21 / 26(80.8 \%)$ & \\
\hline Critical symptoms & 16/102 (15.7\%) & $11 / 76$ (14.5\%) & $5 / 26(19.2 \%)$ & \\
\hline 4C mortality score & 12 IQR (10-14.75) & 12 IQR (10-15) & 12 IQR (10.25-14) & 0.731 \\
\hline \multicolumn{5}{|l|}{ 4C mortality score } \\
\hline Low risk & $1 / 102(1 \%)$ & $1 / 76(1.3 \%)$ & $0 / 26(0 \%)$ & \multirow[t]{4}{*}{0.754} \\
\hline Intermediate risk & 12/102 (11.8\%) & 9/76 (11.8\%) & $3 / 26$ (11.5\%) & \\
\hline High risk & $63 / 102(61.8 \%)$ & $45 / 76(59.2 \%)$ & $18 / 26(69.2 \%)$ & \\
\hline Very high risk & $26 / 102(25.5 \%)$ & $21 / 76(27.6 \%)$ & $5 / 26(19.2 \%)$ & \\
\hline Pulmonary embolism present & $17 / 70(24.3 \%)$ & $12 / 53(22.6 \%)$ & $5 / 17(29.4 \%)$ & 0.746 \\
\hline Deaths & $2 / 102(2 \%)$ & $1 / 76(1.3 \%)$ & $1 / 26(3.8 \%)$ & 0.447 \\
\hline
\end{tabular}

percentages and were compared between the groups using the $\chi^{2}$-test. The ROC curve analysis was used to assess control D-dimer cut-off levels with highest Youden index value for the discrimination of patients with and without the silent DVT. $P$-values $<0.05$ were considered statistically significant. All analyses were performed using the MedCalc statistical software ver. 19.6 (MedCalc Software Ltd, Ostend, Belgium).

\section{Results}

\section{Patient characteristics and features of asymptomatic DVT}

A total of 102 prolonged hospitalized COVID-19 patients without clinical suspicion of DVT were analyzed. The median age was 71.5 years. There was a similar proportion of males $(54 / 102,52.9 \%)$ and females $(48 / 102,47.1 \%)$. A total of $96 / 102(94.1 \%)$ patients required oxygen supplementation due to the COVID-19 pneumonia. According to the previous degree of critical care required, our cohort consisted mostly of prolonged hospitalized ward patients (59/102, 57.8\%), followed by patients separated from the high flow oxygen treatment (HFOT, 33/102, 32.4\%) and mechanically ventilated ICU survivors (10/102, $9.8 \%$ ) admitted to the medical wards after respiratory stabilization. Median length of hospitalization at the time of color duplex ultrasound evaluation was 18 days (median 16, 20 and 19.5 days for ward, HFOT and post-ICU patients, respectively) and median time from the start of COVID-19 symptoms was 27 days.

DVT was detected in a total of 26 out of 102 patients without clinical suspicion (25.5\%). Distal DVT was the most common (22 patients, $84.6 \%$ of the DVT cohort; $21.6 \%$ of the overall cohort). A total of four patients had proximal DVT ( $16 \%$ of the DVT cohort;
$3.9 \%$ of the overall cohort), among them three had thrombosis up to popliteal vein level and one patient had femoral thrombosis. Out of 26 patients 6 (23.1\%) had bilateral leg involvement, 5 with distal and 1 with popliteal DVT. PE was evaluated in a total of 70/102 (68.6\%) of analyzed patients. A total of $17 / 70$ (24.3\%) patients had confirmed PE, and they were similarly distributed among patients with and without asymptomatic DVT $(5 / 17,29.4 \%)$ and $12 / 53,22.6 \%$, respectively; $P=0.571)$. In total $38 / 102(37.2 \%)$ patients included in the study were recognized as having a venous thromboembolism.

\section{Factors associated with presence of asymptomatic DVT}

Patient characteristics stratified according to the presence of asymptomatic DVT are presented in Table 1 and supplementary Table S1. Patients who were mechanically ventilated ICU survivors presented with highest frequency of asymptomatic DVT (6/10, 60\%), whereas patients who were separated from HFOT and prolonged hospitalized patients had similar frequency of asymptomatic DVT $(7 / 33,21.2 \%$ and $13 / 50,22 \%$, respectively) as shown in Fig. 1a. These differences were statistically significant $(P=0.031)$. Intensity of thromboprophylaxis did not significantly differ between patients with and without detected DVT $(P=0.155$; Fig. 1b) and there was no significant difference in preadmission anticoagulation use $(P=0.690)$.

Values for D-dimer were available for 90 patients at the time of hospital admission and for 55 patients as a control measurement. Neither D-dimers at the time of admission to the hospital, nor at the latest control measurement showed significant association with the presence of DVT (Fig. 1c, d); however, there was an evident and statistically significant trend of 

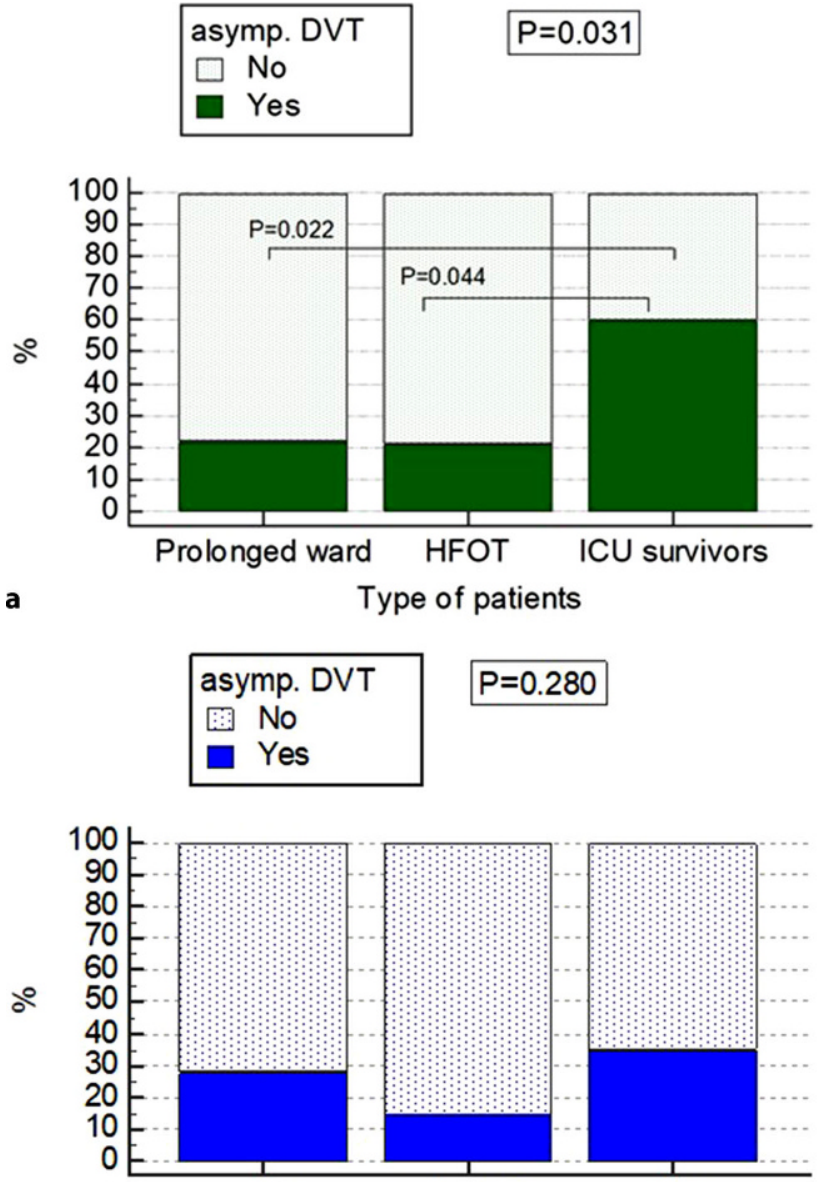

c

Fig. 1 Prevalence of asymptomatic deep vein thrombosis (asymp. DVT) among COVID-19 patients requiring prolonged hospitalization stratified according to the a previous degree of critical care required (prolonged ward hospitalization; sep-

higher frequency of asymptomatic DVT among patients with higher level of control D-dimers $(P=0.034)$. Using the ROC curve analysis we identified control D-dimers cut-off level of $>1.5 \mathrm{mg} / \mathrm{L}$ FEU that was able to stratify patients into two groups (higher and lower control D-dimers) having asymptomatic DVT in $13 / 28(46.4 \%)$ and $5 / 27(18.5 \%)$ patients, respectively; $P=0.027$; however, substantial proportion of prolonged hospitalized COVID-19 patients below this control D-dimer cut-off level, still experienced silent DVT.

Patients with and without detected DVT significantly differed in modified IMPROVE (median 3 and 2 points, respectively; $P=0.021$ ) and IMPROVEDD VTE risk scores (median 4 and 3 points, respectively; $P=0.029$ ) as shown in Fig. 2. Accordingly, asymptomatic DVT was present in 16/45 (35.6\%) patients with $\geq 3$ modified IMPROVE points and 10/57 (17.5\%) with $\leq 2$ modified IMPROVE points; $P=0.038$. It should be noted that only $17 / 26(65.4 \%)$ of patients with silent DVT would fulfil prolonged post-
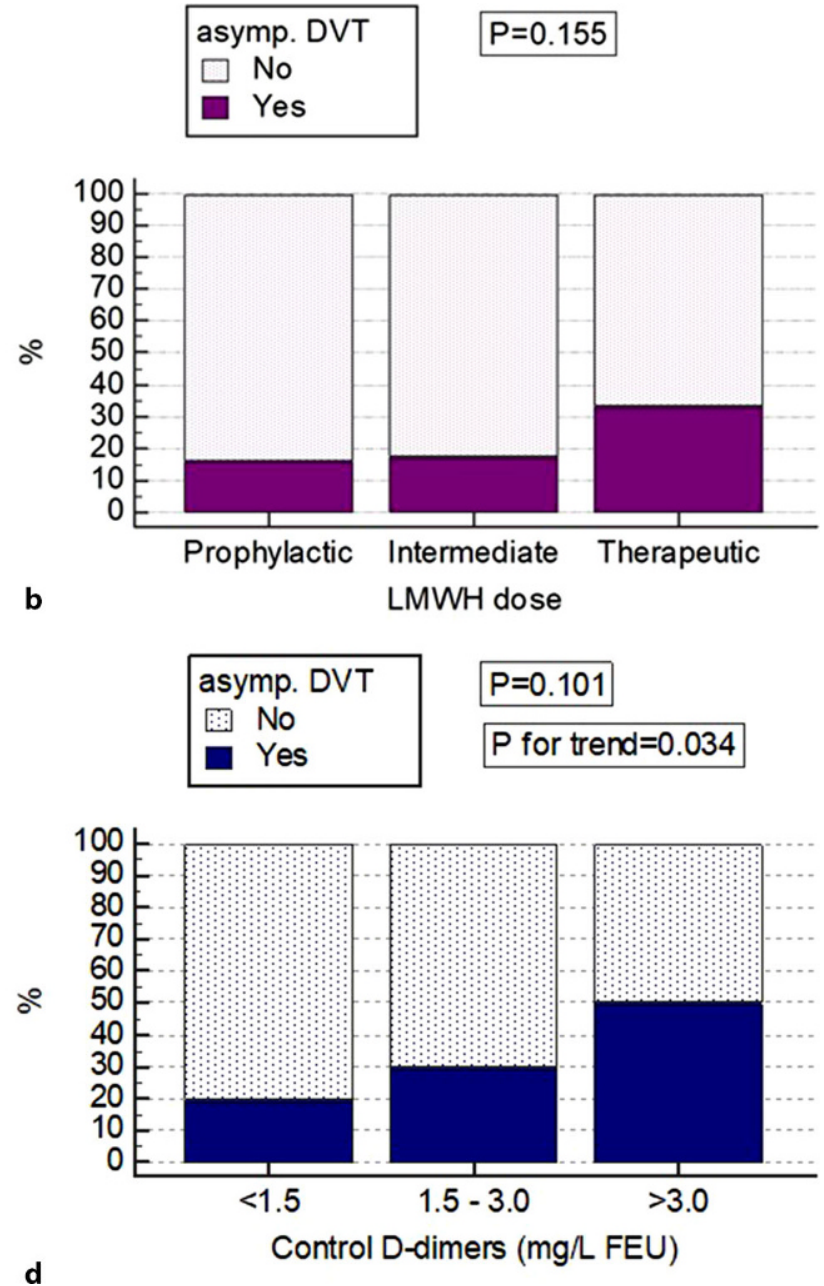

arated from high flow oxygen treatment (HFOT); mechanically ventilated intensive care unit (ICU) survivors), b low molecular weight heparin $(L M W H)$ dosing, $\mathbf{c}$ hospital admission D-dimer levels and $\mathbf{d}$ control D-dimer levels

discharge prophylaxis criteria (modified IMPROVE score of 2 or more with elevated D-dimers $2 \times$ above normal or modified IMPROVE score of 4 or more) [15] in our cohort of patients. Other variables that were significantly associated with the presence of silent DVT were known thrombophilia $(P=0.015)$ and higher body temperature at the time of admission $(P=0.001)$.

Metabolic comorbidities (arterial hypertension, diabetes mellitus, hyperlipidemia, obesity), age, sex, parameters reflecting severity of inflammation (CRP, ferritin, LDH, albumin, WBC, absolute neutrophil and lymphocyte counts, RDW) and clinical prognostic scores describing severity and prognosis of COVID-19 (MEWS, 4C mortality score) were similarly distributed among patients with and without detected DVT. Padua score was not able to predict occurrence of an asymptomatic DVT in our cohort of patients ( $P>0.05$ for all aforementioned analyses). 


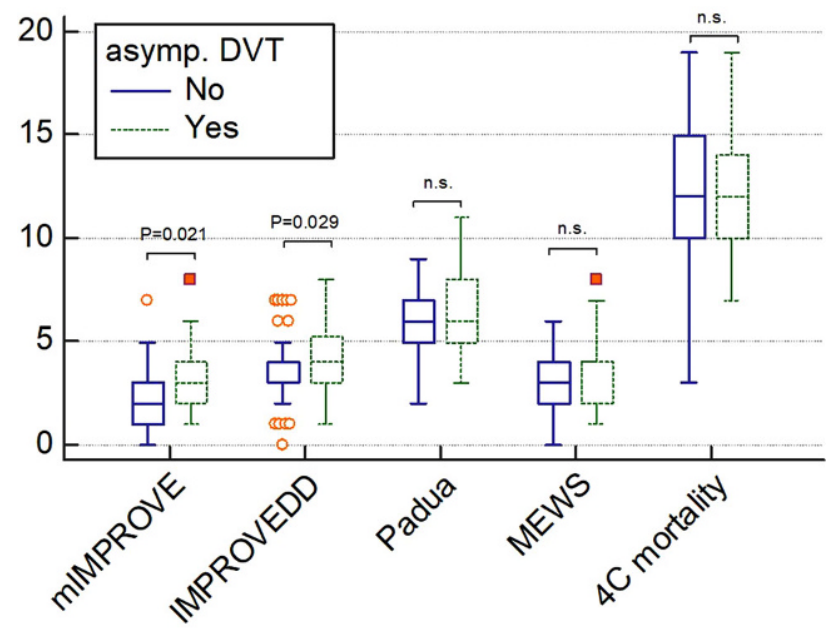

Fig. 2 Comparison of venous thromboembolism prediction scores (modified international medical prevention registry on venous thromboembolism scores [mIMPROVE and IMPROVEDD] and Padua score), and COVID-19 severity scores (modified early warning score [MEWS] and 4C Mortality score) between patients with and without asymptomatic deep vein thrombosis (asymp. DVT). n.s. not statistically significant. Orange/red circles and squares represent outliers as depicted by the statistical program

\section{Discussion}

Due to insufficient data there is no current guideline recommendation either for or against the routine DVT screening in patients without signs or symptoms of VTE [15]. Our data show striking frequency of asymptomatic DVT (25.5\%) in prolonged hospitalized predominantly severe COVID-19 patients despite pharmacologic thromboprophylaxis that was given even in therapeutic LMWH doses in a substantial proportion of patients.

Majority of asymptomatic DVT events detected in our study were distal $(84.6 \%$ of the DVT cohort; $21.6 \%$ of the overall cohort) and unilateral, which are considered to represent low risk of pulmonary embolization if treated. High rate of PE during hospitalization was also detected (24.3\%) which is in line with previous COVID-19 reports [16]. Most interestingly, PE events were similarly distributed among patients with and without asymptomatic DVT. Recommended duration of treatment of VTE is at least 3 months with full intensity anticoagulation. Since all patients are currently exposed to LMWH thromboprophylaxis during hospitalization, clinical relevance of these events probably achieves true extent after the hospital discharge when patients mostly receive no anticoagulation at all or some of them limited duration in reduced doses as a part of prolonged thromboprophylaxis. We do not currently know of any long-term clinical consequences of inadequately treated distal DVT; however, $16 \%$ of asymptomatic events were proximal (3.9\% of the whole cohort) with more pronounced risks of thrombus extension, acute PE and sudden death, chronic thromboembolic pulmonary hypertension, chronic venous insufficiency, etc. Our unpublished experience from post-COVID outpatient clinic shows high prevalence of perfusion defects on pulmonary perfusion scintigraphy consistent with thromboembolism.

Asymptomatic DVT prevalence averaged $21.2 \%$ in ward only patients, $22 \%$ in previous HFOT patients and reached even more concerning $60 \%$ in postmechanically ventilated ICU survivors in the current study. These findings are in line with previous studies routinely conducting bilateral leg ultrasound in hospitalized COVID-19 patients reporting higher than expected DVT prevalence ranging from $11.9 \%$ to $22.5 \%$ in hospitalized ward patients [5-7] and $65-69 \%$ in ICU patients on admission [8, 9]. In all studies investigating ward patients, majority of asymptomatic thromboses were distal and evidently more prevalent than in the landmark MEDENOX trial investigating occurrence of DVT among acutely ill hospitalized non-COVID patients receiving thromboprophylaxis with enoxaparin where incidence of DVT was reported to be $5.5 \%$ (3.8\% for distal and $1.7 \%$ for proximal events) [17]. In contrast to some of these studies [5, 7], we did not observe significant association between admission D-dimers and asymptomatic DVT. In our opinion, this is probably due to longer median time from D-dimer sampling and duplex ultrasound evaluation in our study in comparison to prior studies (median 18 vs. 6 vs. 9 days). Our study confirmed, however, that follow-up D-dimer assessment might improve DVT risk estimation [6] as a significant trend of increase in DVT frequency over rising control D-dimer categories was observable and significant difference in proportion of asymptomatic DVT patients could be observed when patients were stratified at the $>1.5 \mathrm{mg} / \mathrm{L}$ FEU control D-dimer cutoff level. It should be noted that a substantial proportion of these events occurred in the lower control D-dimer group, which speaks in favor of some earlier time of occurrence of these events. Most previous studies could not establish a relationship between the DVT occurrence and laboratory parameters of systemic inflammation or chronic metabolic comorbidities showing that the classical parameters of COVID19 severity cannot help clinicians in recognition of patients with asymptomatic DVT.

Other clinical parameters that were significantly associated with presence of asymptomatic DVT were known thrombophilia, which is included in the modified IMPROVE VTE risk score and higher body temperature at the time of admission, which might indicate higher degree of patient exhaustion and tendency for longer immobilization. Modified IMPROVE score was shown to be significantly higher in patients with than without asymptomatic DVT in our study (median 3 vs. 2 points) and might be useful tool for clinical orientation. Unfortunately, Padua risk score did not show 
good predictive properties neither in our nor previous studies [5].

It should be noted that current COVID-19 guidelines do not recommend routine intermediate or therapeutic intensity anticoagulation over prophylactic dosing in hospitalized patients [18]; however, guidelines allow to consider increasing the intensity of anticoagulation, especially in critically ill patients, and this is an everyday real-life clinical practice as our data confirm. It is unclear, however, how much intermediate and therapeutic LWMH doses reduce the incidence of DVT (no significant difference in DVT frequency was found between patients on different LMWH thromboprophylaxis doses in the current study) and if they can actually paradoxically silent the clinical presentation and allow for increased number of undetected DVT patients to be discharged from hospital. Majority of patients in the current study had pneumonia requiring oxygen supplementation and were receiving corticosteroids where the antiinflammatory properties might also contribute to the lack of DVT clinical presentation.

Routine post-discharge thromboprophylaxis is not recommended in unselected COVID-19 patients [15]; however, patients who are currently considered that may benefit from post-discharge thromboprophylaxis are ones with modified IMPROVE VTE risk score $\geq 4$ or modified IMPROVE VTE risk score $\geq 2$ and D-dimer levels $>2$ times the upper normal limit $[15,19]$. It should be noted that a substantial proportion of patients in our study would be discharged from hospital with unrecognized DVT without fulfilling criteria for extended thromboprophylaxis (35\% of DVT patients). Also, DOAC reimbursement issues are affecting the decision to anticoagulate a patient after hospital discharge even if the patient fulfils the extended thromboprophylaxis criteria without proven VTE, further emphasizing the importance of establishing a diagnosis with objective imaging methods.

Limitations of our work are single center experience, relatively small number of patients and inability to determine the exact time of DVT occurrence. Nevertheless, our findings confirm previous reports of high prevalence of asymptomatic DVT and call for reconsideration of guideline recommendations for routine DVT screening in asymptomatic patients, especially in ICU survivors and patients with IMPROVE score $\geq 3$.

In summary, our study systematically performing bilateral leg color duplex ultrasound in prolonged hospitalized COVID-19 patients without clinical suspicion of DVT found high prevalence $(25.5 \%)$ of mostly distal asymptomatic DVT. Asymptomatic DVT events were more common in ICU survivors, patients with higher modified IMPROVE score and higher body temperature on admission. No clear associations with demographic and other clinical characteristics, intensity of thromboprophylaxis, severity of COVID-19 symptoms, degree of systemic inflammation or D-dimers on ad- mission were found. PE was also highly prevalent (24.3\%) but similarly grouped among patients with and without asymptomatic DVT. Systematic ultrasound assessment of prolonged hospitalized severe COVID-19 patients prior to hospital discharge is probably needed to timely recognize and appropriately treat patients with asymptomatic DVT.

\section{Declarations}

Conflict of interest M. Lucijanic, N. P. Zivkovic, M. Ivic, M. Sedinic, B. Brkljacic, A. Mutvar, D. Rudan, B. Barsic, I. Luksic, R. Kusec and G. Ivanac declare that they have no competing interests.

Ethical standards All procedures performed in studies involving human participants or on human tissue were in accordance with the ethical standards of the institutional and/or national research committee and with the 1975 Helsinki declaration and its later amendments or comparable ethical standards. Informed consent was obtained from all individual participants included in the study.

\section{References}

1. Wu Z, McGoogan JM. Characteristics of and important lessons from the coronavirus disease 2019 (COVID-19) outbreak in China: summary of a report of 72314 cases from the Chinese Center for Disease Control and Prevention. JAMA. 2020;323(13):1239-42.

2. Panigada M, Bottino N, Tagliabue P, et al. Hypercoagulability of COVID-19 patients in intensive care unit: a report of thromboelastography findings and other parameters of hemostasis. JThromb Haemost. 2020;18(7):1738-42.

3. Terpos E, Ntanasis-Stathopoulos I, Elalamy I, et al. Hematological findings and complications of COVID-19. Am J Hematol. 2020;95(7):834-47.

4. Bilaloglu S, Aphinyanaphongs Y, Jones S, Iturrate E, Hochman J, Berger JS. Thrombosis in hospitalized patients with COVID-19 in a New York City health system. JAMA. 2020;324(8):799-801.

5. Santoliquido A, Porfidia A, Nesci A, et al. Incidence of deep vein thrombosis among non-ICU patients hospitalized for COVID-19 despite pharmacological thromboprophylaxis. J Thromb Haemost. 2020;18(9):2358-63.

6. Artifoni M, Danic G, Gautier G, et al. Systematic assessment of venous thromboembolism in COVID-19 patients receiving thromboprophylaxis: incidence and role of D-dimer as predictive factors. J Thromb Thrombolysis. 2020;50(1):211-6.

7. Demelo-Rodríguez P, Cervilla-Muñoz E, Ordieres-Ortega L, et al. Incidence of asymptomatic deep vein thrombosis in patients with COVID-19 pneumonia and elevated D-dimer levels. Thromb Res. 2020;192:23-6.

8. Nahum J, Morichau-Beauchant T, Daviaud F, et al. Venous thrombosis among critically ill patients with coronavirus disease 2019 (COVID-19). JAMA Netw Open. 2020;3(5):e2010478.

9. Llitjos JF, Leclerc M, Chochois C, et al. High incidence of venous thromboembolic events in anticoagulated severe COVID-19 patients. J Thromb Haemost. 2020;18(7):1743-6.

10. Raskob GE, Spyropoulos AC, Zrubek J, et al. The MARINER trial of rivaroxaban after hospital discharge for medical patients at high risk of VTE. Design, rationale, and clinical implications. Thromb Haemost. 2016;115(6):1240-8. 
11. Gibson CM, Spyropoulos AC, Cohen AT, et al. The IMPROVEDD VTE risk score: incorporation of D-dimer into the IMPROVE score to improve venous thromboembolism riskstratification. THOpen. 2017;1(1):e56-e65.

12. Barbar S, Noventa F, Rossetto V, et al. A risk assessment model for the identification of hospitalized medical patients at risk for venous thromboembolism: the Padua Prediction Score. JThromb Haemost. 2010;8(11):2450-7.

13. Subbe CP, Kruger M, Rutherford P, Gemmel L. Validation of a modified Early Warning Score in medical admissions. QJM. 2001;94(10):521-6.

14. Knight SR, Ho A, Pius R, et al. Risk stratification of patients admitted to hospital with covid-19 using the ISARIC WHOClinical Characterisation Protocol: development and validation of the 4C Mortality Score. BMJ. 2020;370:m3339.

15. COVID-19 Treatment Guidelines Panel. Coronavirus disease 2019 (COVID-19) treatment guidelines. 2021. https:// www.covid19treatmentguidelines.nih.gov/. Accessed 2 Feb 2021, National Institutes of Health.

16. Grillet F, Behr J, Calame P, Aubry S, Delabrousse E. Acute pulmonary embolism associated with COVID-19 pneumo- nia detected with pulmonary CT angiography. Radiology. 2020;296(3):E186-E8.

17. Samama MM, Cohen AT, Darmon JY, et al. A comparison of enoxaparin with placebo for the prevention of venous thromboembolism in acutely ill medical patients. Prophylaxis in medical patients with enoxaparin study group. NEngl J Med. 1999;341(11):793-800.

18. FlaczykA, RosovskyRP, Reed CT, Bankhead-Kendall BK, BittnerEA, ChangMG.Comparison of published guidelines for management of coagulopathy and thrombosis in critically ill patients with COVID 19: implications for clinical practice and future investigations. CritCare. 2020;24(1):559.

19. Spyropoulos AC, Lipardi C, Xu J, et al. Modified IMPROVE VTE risk score and elevated D-dimer identify a high venous thromboembolism risk in acutely ill medical population for extended thromboprophylaxis. TH Open. 2020;4(1):e59-e65.

Publisher's Note Springer Nature remains neutral with regard to jurisdictional claims in published maps and institutional affiliations. 